\title{
Psychological experiences of nurses in COVID-19 isolation wards in China: A qualitative examination
}

\author{
Guoguo Chen ${ }^{1}$, Ping Zou*2, Honghua Zhou ${ }^{3}$, Xiaowen Shen ${ }^{3}$, Chenchen Gao ${ }^{4}$, Jinhong Ying ${ }^{3}$, Sabrina L. Balkaran ${ }^{5}$, \\ Chunmei Zhang ${ }^{\dagger 4}$ \\ ${ }^{1}$ The Second Affiliated Hospital of Wenzhou Medical University, Wenzhou, Zhejiang, China \\ ${ }^{2}$ School of Nursing, Nipissing University, Toronto, Ontario, Canada \\ ${ }^{3}$ Second School of Clinical Medicine, Wenzhou Medical University, Wenzhou, Zhejiang, China \\ ${ }^{4}$ School of Nursing, Wenzhou Medical University, Wenzhou, Zhejiang, China \\ ${ }^{5}$ McMaster University, Hamilton, Ontario, Canada
}

Received: August 18, 2020

DOI: $10.5430 /$ jnep.v11n2p56
Accepted: September 27, $2020 \quad$ Online Published: October 25, 2020

URL: https://doi.org/10.5430/jnep.v11n2p56

\begin{abstract}
Objective: This study explores the psychological experiences and factors affecting the psychological health of isolation ward nurses during the COVID-19 pandemic in China. Findings from this study will inform on future initiatives that can support frontline nurses, improve their ability to cope with public health emergencies, and create effective nursing management strategies. Methods: A qualitative phenomenological research method was applied. In-depth interviews with 10 first-line clinical nurses in isolation wards during the COVID-19 pandemic were conducted. Collected data were analyzed with the Colaizzi seven-step analysis method.

Results: Three themes were emerged, including the evolution of the psychological health of nurses, positive factors that affect psychological health, and negative factors that affect psychological health. Nurses experienced various changes to their psychological health during the pandemic. When nurses were supported by team members, appreciated by their patients, and cared for by their organizations, their psychological health was enhanced. When nurses felt disconnected from their families and experienced physical discomfort due to protective equipment, their psychological health was negatively impacted.

Conclusions: Changes in the psychological health of isolation ward nurses should be constantly monitored. Proper strategies should be implemented to reduce the physical discomfort experienced by nurses due to protective equipment, increase perceptions of familial and workplace support, and determine ways to increase displays of patient appreciation for nursing work.
\end{abstract}

Key Words: COVID-19, Nurses, Psychological experience, Isolation wards, Qualitative research

\section{INTRODUCTION}

A coronavirus disease 2019 (COVID-19) pandemic has been declared by the World Health Organization. As of June 2020, there are over 11 million cases and over 500,000 fatalities worldwide, with 85,306 confirmed cases and 4648 fatalities in China alone. ${ }^{[1]}$ China has responded to the high incidence rate of COVID-19 with aggressive social distancing policies, public education campaigns and social media campaigns. ${ }^{[2]}$ The Lunar New Year holiday was extended to decrease inter-

\footnotetext{
*Correspondence: Ping Zou; Email: pingz@ nipissingu.ca; Address: School of Nursing, Nipissing University, 750 Dundas Street West, Room 209, Toronto, Ontario, Canada.

† Correspondence: Chunmei Zhang; Email: sallyzcm@126.com; Address: School of Nursing, Wenzhou Medical University, Wenzhou, Zhejiang,
} China. 
provincial travelling, which helped to control rising COVID19 cases. ${ }^{[3]}$ Daily reports creating epidemiological curves for the country and each province were generated through China's National Infectious Disease Information System to track the characteristics of affected demographics, progression and zoonotic origins of COVID-19. ${ }^{[4]}$ Hospitals have taken swift action against the pandemic, with China mobilizing over 40000 medical personnel to limit the spread of COVID-19. In addition, two new hospitals, the Huoshenshan Hospital and the Leishenshan Hospital were built specifically for the treatment and containment of patients diagnosed with COVID-19. ${ }^{[4]}$

Wenzhou is a well-developed industrial and commercial city in southeast China with a large national migratory population. In Wenzhou, there are around 330,000 workers from Hubei province, which is the epicenter of the COVID-19 pandemic in China. In addition, Wenzhou businessmen travel throughout the country, with around 180,000 currently residing in Hubei province. Wenzhou had the greatest number of confirmed cases in Zhejiang province, and was one of the most affected cities outside Hubei province. The municipal government issued a quarantine lockdown to actively respond to the pandemic. Under the guidance of the government, hospitals in Wenzhou created new floors for the specialized treatment of COVID-19 patients. Nursing departments deployed nurses from all pre-existing wards to form new nursing teams that worked on specialized COVID19 treatment floors. During this unexpected and dangerous pandemic, the nursing teams had to adapt to not only the intensive nursing work, but also had to implement novel COVID-19 protective equipment. In addition, nurses were separated from their families for an extended period of time, making them feel disconnected and lonely. Therefore, nurses were a subset of the medical population that were extremely vulnerable to adverse psychological experiences. ${ }^{[5,6]}$

The maintenance of a positive psychological state among healthcare workers is not only critical to their mental health, but also to the quality of care received by their patients. In hospitals, healthcare workers have previously cited underpayment, work overload, and inadequate staffing and management issues as the primary sources of stress and negative psychological health. ${ }^{[7,8]}$ Levels of distress among healthcare workers during infectious outbreaks such as the Severe Acute Respiratory Syndrome (SARS) pandemic of 2003 were significantly higher and were primarily attributed to the loss of control over their own health, fear for their own health, and spread of the SARS virus. ${ }^{[9,10]}$ Healthcare workers also worried that protective equipment may provide insufficient protection against the disease. ${ }^{[11]}$ The psychological states

Published by Sciedu Press of healthcare workers have been found by previous literature to have a direct impact on patient quality of care and patient safety. ${ }^{[12,13]}$ Healthcare professionals experiencing higher levels of anxiety and burnout were found to make more medical errors and have more lapses in professional judgement, which can severely compromise patient care and patient health. ${ }^{[13-16]}$

Nurses are a demographic that are particularly susceptible to experiencing poor psychological health due to the heightened frequency and length of their interactions with patients. ${ }^{[11]}$ Studies evaluating the psychological impacts on frontline healthcare workers during the SARS pandemic of 2003 found that nurses experienced higher levels of stress and psychological distress compared to other healthcare professionals. ${ }^{[10,11]}$ Thus, it is imperative that we consider the psychological health of nurses during the COVID-19 pandemic to ensure both the safety of the nurses, and the patients that they care for. In this study, a phenomenological research approach to qualitative research was used to explore the psychological experiences of nurses in the isolation ward during the COVID-19 pandemic in Wenzhou city, Zhejiang Province, China, and explore factors affecting the psychological health of nurses. Study findings will inform on future initiatives to support frontline nurses, improve their capability to cope with public health emergencies, and create effective nursing management strategies.

\section{METHODS}

\subsection{Ethical considerations}

The research ethics approval was obtained from a university research ethics review board in China. All participants were fully informed about the purpose of the study and informed consent was received for the recording of interviews.

\subsection{Design and sampling}

In this study, a purposeful sampling method was used to select first-line nurses in Wenzhou who were specifically involved in the treatment of patients with COVID-19. Nurses were included if they participated in the response of public health emergencies and took care of COVID-19 patients directly in a COVID-19 isolation ward. The sample size was set to interview another two participants once the emerging themes from the collected data repeat and no new theme appears at the time of data analysis. A total of 10 nurses were interviewed in this study.

\subsection{Participant demographics}

A total of 10 frontline nurses ( 8 females and 2 males) were recruited in this study (see Table 1). The majority of participants originally worked in the departments of Internal 
Medicine (4.40\%) and Surgery (4.40\%). Two participants were under 30 years of age, five participants were between 30-35 years of age, and three participants were over 35 years of age. Most participants were married (8.80\%), had children
(7.70\%), had bachelor's or higher degrees (9.90\%), and had at least 5 years of work experience as professional nurses (9.90\%). Most participants worked in COVID-19 isolation wards for at least 7 days $(7.70 \%)$.

Table 1. General information of interviewees $(n=10)$

\begin{tabular}{|c|c|c|c|}
\hline Variable & Category & $\mathbf{n}$ & $\%$ \\
\hline \multirow{4}{*}{ Department } & Internal Medicine & 4 & 40 \\
\hline & Surgery & 4 & 40 \\
\hline & Obstetrics and Gynecology & 1 & 10 \\
\hline & Pediatrics & 1 & 10 \\
\hline \multirow{2}{*}{ Gender } & Man & 2 & 20 \\
\hline & Woman & 8 & 80 \\
\hline \multirow{3}{*}{ Age } & $<30$ & 2 & 20 \\
\hline & 30 to 35 & 5 & 50 \\
\hline & $>35$ & 3 & 30 \\
\hline \multirow{4}{*}{ Marital status } & Single & 2 & 20 \\
\hline & Married & 8 & 80 \\
\hline & Widowed & 0 & 0 \\
\hline & Separated & 0 & 0 \\
\hline \multirow{2}{*}{ Childbearing status } & Yes & 7 & 70 \\
\hline & No & 3 & 30 \\
\hline \multirow{3}{*}{ work experience (years) } & $<5$ & 1 & 10 \\
\hline & 5 to 10 & 5 & 50 \\
\hline & $>10$ & 4 & 40 \\
\hline \multirow{3}{*}{ Educational level } & Diploma/Certificate & 1 & 10 \\
\hline & Bachelor’s degree & 8 & 80 \\
\hline & Master degree & 1 & 10 \\
\hline \multirow{3}{*}{ Job title } & Nurse & 6 & 60 \\
\hline & Supervisor nurse & 3 & 30 \\
\hline & Co-chief nurse & 1 & 10 \\
\hline \multirow{3}{*}{$\begin{array}{l}\text { Combating the epidemic } \\
\text { service (days) }\end{array}$} & $<7$ & 3 & 30 \\
\hline & 7 to 14 & 6 & 60 \\
\hline & $>14$ & 1 & 10 \\
\hline
\end{tabular}

\subsection{Data collection}

The interview outline was created after consulting the relevant literature and expert consultations. ${ }^{[17,18]}$ Interview questions were: (1) How did you feel when you were called to work on the COVID-19 clinical front lines? (2) How did you feel when treating patients with COVID-19? (3) What impressed you while working in the isolation ward? (4) What are your needs during the pandemic? The interviewer was a first-line nurse working in isolation wards of COVID-19 and completed a comprehensive systematic study of qualitative research methods and interview techniques before the study. The interviewer, along with the nurses that were interviewed were frontline clinical nurses treating COVID-19 patients, which facilitated communication and allowed them to relate to each other. Before an interview, participants were informed of the purpose, meaning, anonymity and confiden- tiality of the study. The interviews were conducted in an independent small room in the living area of the designated specialized hospital. During the interview process, the small room was quiet and was not disturbed by the outside world. The interviewer obtained the participant's informed status and consent before commencing the synchronized recording. Interviews lasted approximately 20 to $40 \mathrm{~min}$.

\subsection{Data analysis and quality control}

After the interview, the researcher listened carefully to the recording, transcribed it, and coded it word for word. ${ }^{[19]}$ Due to ethical and moral considerations, after the transcription was completed, the interview recording was deleted after confirming that it was no longer required for analysis. ${ }^{[19]}$ The transcribed interview manuscript was then returned to the participant for review, and inaccurate points of the transcrip- 
tion were corrected to ensure the integrity of the data. The data was then analyzed by Colaizzi's 7-step analysis method, which required researchers to: (1) carefully read all interview records; (2) analyze and extract important statements; (3) encode the repeated meaningful viewpoints; (4) assemble the coded viewpoints; (5) write a detailed and complete description; (6) distinguish similar viewpoints and extract thematic concepts; and (7) return to the participants for verification. ${ }^{[20]}$ Two researchers that were familiar with qualitative research conducted the data analysis independently, and then data analysis results were compared and contrasted. Any inconsistencies were resolved through discussion among the research team.

\section{RESUlTS}

Ten interviews were conducted with front line nurses that worked in isolation wards. When interview transcripts were evaluated with the Colaizzi 7-step analysis, three main themes emerged: the evolution of psychological health throughout the pandemic, factors that positively affect psychological health, and factors that negatively affect psychological health. Our results section will delineate these three themes and their respective subsections.

\subsection{Evolution of the psychological health of nurses}

Participants revealed that their psychological health had gradually changed during the COVID-19 Pandemic. At the onset of the pandemic, they reported being in a poor psychological state due to fear of the virus and of being separated from their families. After entering isolation wards to start treating COVID-19 cases, they gradually adjusted to the work rhythm and environment. After this adjustment, the psychological health of the nurses gradually stabilized. Towards the end of the pandemic, nurses working in isolation wards realized the importance of their work, which brought them a sense of professional pride and a sense of achievement. This positive realization was reflected in an increase in the psychological health of frontline nurses.

\subsubsection{Onset of the pandemic}

During the pandemic, every nurse in the isolation ward was actively involved in the treatment of patients. However, COVID-19 is a novel coronavirus, and due to the lack of knowledge surrounding its causes, transmission and potency, nurses were afraid of being infected and of causing harm to their colleagues and family. Each participant mentioned during interviews that they experienced contradictory feelings and felt lost when they were inducted as frontline COVID-19 workers. A nurse shared, "I am a nurse. When the epidemic broke out, it's my unavoidable responsibility to work on the front lines. However, this time, the novel coronavirus was highly contagious, and I was afraid of being infected (Nurse 8)." Another nurse expressed her contradictory feelings about the COVID-19 pandemic: "When I received the notice, I was very excited. It was the first time for me to participate in a public health emergency; but I was afraid that I would not do well because of my lack of knowledge, which may burden the team (Nurse 10)." Similar concerns were expressed among other nurses, with another participant mentioning: "Although I am very afraid that I might become ill, the patients infected with COVID-19 are more helpless than I am. Since I can do something, I should help them to fight their disease (Nurse 10)."

\subsubsection{Adaptation period}

Some participants said they had adapted to the new working environment during their first day of work in the isolation ward without experiencing fear, anxiety, or unease. Other participants said that their negative experiences only deceased after working in the isolation ward for a period of time. One participant mentioned: "I remember when I was putting on protective gear outside the isolation ward, I was still nervous; when I really went in, I calmed down and began to adapt to the work (Nurse 2)." Another nurse revealed: "I forgot my initial fear when I was facing a confirmed COVID-19 case in my isolation jacket. I realized that I was a nurse, that the patient needed me, and that I should take good care of them (Nurse 5)." Another participant expressed: "Before long after I went in, I began to feel nauseous for over 2 hours; during that period, I thought about quitting early, but then I gradually recovered from that bad feeling (Nurse 4)." An additional participant mentioned: "I have worked in the isolation ward for some time and am relatively familiar with the workload, so I adapted very well to the rhythm of work here. There was no more anxiety in my mind (Nurse 10)." Another nurse said: "I am walking back and forth between the living area and the working area every day; this life is not so bad for me; I always think about how I can improve my efficiency from time to time when I have spare time (Nurse 1)." An interviewed nurse also mentioned: "When I entered the quarantine ward, I found that except for wearing protective equipment, our work here was not so different from that of a general ward. Although it is clumsy to work in this way, the overall process is quite smooth. When I think back, my previous depressed mood was not necessary at all (Nurse 3)."

\subsubsection{Later phases of the pandemic}

As the pandemic progressed, the psychological health of most frontline clinical nurses stabilized. They gradually recognized themselves as COVID-19 frontline workers and experienced high levels of job satisfaction as professional nurses. A nurse mentioned: "To the public, nurses no longer only handle injections and medicines. They now call us 
the most beautiful angels in the fight against the pandemic (Nurse 2)." Another participant expressed: "Now the public has realized that we are making a fundamental contribution to society, and our status in the eyes of the public has been enhanced (Nurse 10)." An interviewed nurse also mentioned: "I was the first one in our department to apply for an extension of the work cycle because I wanted to see my patients fully recover and leave the hospital, so I would feel fulfilled in my job (Nurse 8)." Another nurse mentioned "If I have to work on the front line again, I would definitely sign up because this experience has allowed me to reflect on my professional values and discover a sense of pride that I have never experienced before (Nurse 1)."

\subsection{Factors positively affecting psychological health}

\subsubsection{Appreciation from patients}

Although working in an isolation ward is emotionally taxing, interviewed nurses felt a sense of achievement and professional responsibility when being appreciated and encouraged by their patients. These factors promoted positive psychological health among nurses that helped them to cope with the pandemic. One participant noted: "The little girl in bed 29 folded paper hearts for me, showing her respect. She not only drew pictures on it, but also thanked us. I was deeply touched at that moment (Nurse 3)." Another nurse mentioned: "Every time we go into the ward, the patients always tell us 'thank you very much, you nurses are angels for us, you are really great.' I didn't hear such moving remarks very often before, and I felt very proud (Nurse 1). Another nurse said: "A pregnant woman invited us to take a picture with us before leaving the hospital. She said that she would miss us in the future, and she would remember our meticulous care for her whole life. We were so touched and happy that we were moved to tears (Nurse 7)."

\subsubsection{Support from one's team}

Support from other team members helped to increase positive psychological health among nurses. Nurses helped, comforted, and encouraged each other to help manage their negative emotions and physical discomfort. One nurse mentioned: "Although we are in such a difficult situation, I am grateful that I could become a member of this team because everyone laughs and cries together (Nurse 6)." Another participant said "I experienced discomfort while menstruating, and one of my companions immediately give me her own ginger tea with brown sugar. In this way, we always take care of each other in a timely manner (Nurse 4)." A participant expressed: "It was a nurse's birthday, so we wrote 'happy birthday' for her by using test tubes of different colors, and also took photos of her (Nurse 5)." Another nurse mentioned "The department is full of kindness, especially from our head nurse. To guarantee the preventive effect of Chinese medicine, our head nurse allowed everyone to drink, and the canteen staff was specifically ordered not to add radish to our diet during this time as it may reduce the medicinal effects. She really cares and is detail-oriented (Nurse 2)."

\subsubsection{Feeling cared for and supported by the organization}

Participants reported that the leaders, the union, and the logistics department effectively supported them, which helped them to manage their negative moods. One nurse mentioned: "The nursing department often asks about our needs, replenishes supplies and food in a timely manner, and keeps an eye on our health (Nurse 2)." Another participant expressed: "The canteen staff guarantees our nutritional needs every day and offer us extra dishes without additional charge (Nurse 3)." Another participant also mentioned: "If we apply to receive any medication from the union, it will be delivered the next day (Nurse 4)." Another nurse expressed: "The hospital designated someone to be in charge of our daily activities in our living area, and since then, small life problems are now solved in a timely manner (Nurse 9)." One nurse also noted: "We are a new team, but there is strong leadership and our entire team gets along very well (Nurse 5)."

\subsection{Factors negatively affecting psychological health 3.3.1 Perceived familial disconnect}

Due to the intensive workload as frontline workers, isolation ward nurses worked long hours and were ultimately separated from their families due to rigorous quarantining processes. As result of this prolonged familial separation, nurses felt disconnected and distressed. One nurse expressed: "When I was tidying up and preparing to come over, my daughter kept asking me to stay because she missed me. At that time, my heart was very heavy. Now when I think of that time, my heart always aches faintly because I don't see her enough (Nurse 7)." One participant said: "My mother is the one that I think about the most while working on the front lines. She has been not sleeping well, and now she worries about my safety every day. I'm really afraid for her wellbeing (Nurse 4)." Another nurse expressed: "These days, my husband has been complaining that he is going to collapse. Now he is taking care of our two children at home all by himself. His parents in his hometown can't come over because of the village closure. The nanny can't come either. Our youngest child is less than 2 years old and wakes up at 4 o'clock in the morning. We also have to help our elder child prepare for online study and supervise him when he completes his homework. I miss my children and can only wipe my tears away silently (Nurse 9)."

Some participants were afraid they were causing their families undue stress because they were worrying about their 
health as front line nurses. Front line nurses were not only missing their children, but they worried about the safety of their families. These combined stresses caused their emotional states to drastically fluctuate, which made them prone to experiencing poor psychological health. One nurse mentioned "Since my mother knows that I am a nurse fighting COVID-19, she sent me many WeChat messages every day. She would not be relieved until she saw my replies informing her I was safe. I am really ashamed that I still worry my parents. I will not let them down, I will work hard, and I will make them proud (Nurse 2)."

\subsubsection{Discomfort from protective equipment}

Isolation ward nurses reported that the protective equipment was uncomfortable, which made them feel nervous, uneasy and even irritated. These negative feelings greatly contributed to their poor psychological health. One nurse said: "I'm wearing protective clothing and wearing two masks, which makes me feel very stuffy. I always need to breathe heavily, and the N95 mask is either hurting my nose, or is clamping my nose wing so I can't breathe properly. Dealing with long-term hypoxia makes me feel very anxious (Nurse 4)." Another participant mentioned: "On one occasion, I entered the isolation ward and felt particularly stuffy. As time progressed, I was feeling a little nauseous, and very uneasy (Nurse 5)." Another participant said: "Although my face is pasted with hydrocolloids, my cheeks suffered acute pain because of the goggles and N95 masks. In the isolation ward, the pain on my face increasingly occupied my mind (Nurse 8)." One nurse said: "My goggles often become foggy, and my inability to see increased the difficulty of my work. For example, when facing the computer, I could not write my nursing records. The goggles also caused me to experience venous blood collection and punctures (Nurse 3)."

\section{DisCUSSION AND RECOMMENDATIONS}

\subsection{Address the fluctuating psychological health of nurses}

\subsubsection{Acknowledge the psychological health of nurses at all stages}

Our study findings suggested that nurses experience various changes in their psychological health while working in isolation wards. Towards the beginning of the pandemic, the psychological health of nurses working on the COVID-19 frontlines was poor because were afraid of contracting the virus, falling ill, and transmitting it to their loved ones. These findings are consistent with previous studies during the SARS outbreak. ${ }^{[21]}$ Nurses experienced a gradual psychological adaptation during the SARS outbreak as they demonstrated a significant reduction in poor mood ratings, insomnia and negative feelings at the pandemic progressed. ${ }^{[21]}$ The anxiety Published by Sciedu Press and poor psychological health of nurses in the early stages of the pandemic were caused by fear of the unknown disease, and external pressure. ${ }^{[10,22]}$

It is recommend that nursing managers guide and care for frontline nurses during this period and provide them with COVID-19-related information. Most frontline nurses in our study reported that the paucity of information surrounding COVID-19 made them feel anxious because they were afraid of being infected, falling ill, and transmitting the virus to their loved ones. Being informed of the origins, and methods of transmission of COVID-19 could reduce their trepidation by increasing their confidence in their protective equipment, and in their own abilities to sanitize and prevent transmission to their families and friends. Previous studies have established that during the SARS pandemic, nurses reported having a greater understanding of SARS-related knowledge towards the end of the outbreak, which helped them cope and feel more secure in their work. ${ }^{[21]}$ As such, we recommend that nursing managers keep front lines nurses abreast of the latest COVID-19 information, because it will help stabilize their psychological health. Moreover, many nurses in our study reported feeling lonely and disconnected from their families, which negatively affected their psychological health. While nurses adapt to their new circumstances, nursing managers should identify changes in their psychological health over time. By identifying these changes, nursing managers can assess how and when to best support, guide, and care for frontline nurses. If front line nurses feel supported when they are at their loneliest due to familial separation, a new sense of community could be established which could ameliorate their psychological health.

As the pandemic progressed, nurses adapted to the new intensive work conditions, and their psychological health stabilized. Later on, nurses reported developing a sense of mission and professional achievement. This realization enhanced their professional values, which suggests that nurses are more devoted to their jobs after large-scale public health events. In accordance with our findings, other studies also suggested that while a significant level of negative emotions was reported in the early stages of the COVID-19 pandemic, healthcare workers employed a number of self-coping mechanisms which increased their gratefulness, professional responsibility and self-reflection as the pandemic progressed. ${ }^{[22]}$

\subsubsection{Address the psychological health of nurses during different phases of the pandemic}

Establishment of a psychological health support team for isolation ward nurses is recommended. This psychological support team would consist of senior nurses specializing in psychology that had counselors' qualifications. The psycho- 
logical support team would conduct regular psychological evaluations on nurses working in isolation wards, ascertain psychological problems experienced by nurses, and provide one-on-one psychological interventions.

The creation of psychological health support teams will help to address the fluctuating emotional and psychological health of frontline nurses during the COVID-19 pandemic. Our findings showed that nurses were most vulnerable at the onset of the pandemic, experiencing symptoms of anxiety because they felt isolated from their families, and were fearful of contracting and potentially transmitting the virus. Psychological health teams could help address these concerns as they emerge, thereby preventing further harm to frontline nurses. Similar strategies have been implemented during the SARS outbreak. ${ }^{[23]}$ For example, nurses received a group session therapy intervention where they discussed their emotional stress. Emotional support helped nurses manage their emotions, feel accepted, and realize that they were not alone. ${ }^{[23]}$ Existing studies have indicated that effective psychological interventions can help resolve psychological crises experienced by nurses. ${ }^{[24,25]}$ If nurses in isolation wards experience high levels of mental stress, it may become increasingly difficult for them to manage their negative emotions, which may affect their work and ability to provide quality patient care. ${ }^{[12]}$ Nurses could also seek help from counselors in the psychological support team to obtain targeted and professional guidance to help them tackle work challenges and develop a positive mental state.

\subsubsection{Enhance communication between nursing man- agers and frontline nurses}

Our study findings revealed that when nurses feel supported by their managers and their organization, their psychological health is positively affected. Due to their heavy workload, team managers may at times have limited communication with nurses working in isolation wards, which negatively impacts the nurses' trust and working efficiency. ${ }^{[26]}$ Managers should consistently communicate with nurses to evaluate the changes in their psychological health during different stages of the pandemic, and provide guidance to them. Enhancing the communication between nursing managers and frontline nurses will help improve the psychological state of nurses. Studies have previously suggested that leadership and positive praise can increase nurses' motivation to work. ${ }^{[27]}$ When delegating staff, managers can implement "the Experienced and Junior Matching model", in which experienced isolation ward nurses help junior nurses manage their negative emotions based on their personal experience, and familiarize junior nurses with the isolation ward workflow. Team managers should check in with nurses from time to time each week, extend their appreciation for the nurses' hard work, and understand the difficulties faced by nurses.

\subsection{Enhance positive factors affecting psychological wellbeing}

\subsubsection{Improve communication between patients and} nurses

Effective communication between nurses and patients has been shown to improve the quality of care and increase positive outcomes among patients. ${ }^{[28]}$ In our study, frontline nurses found that when they felt appreciated by their patients, they worked harder and experienced better psychological health. Previous studies align with our findings, indicating that patient recognition of nursing work can decrease the rate of nurse burnout, which can consequently increase the patient's satisfaction with the care that they receive. ${ }^{[29]}$ When being treated, patients often only see the nurse's eyes when communicating face to face. Nurses should pay attention to eye contact when communicating with patients at close range, so that patients can feel heard and understood. By improving nursing communication skills, patients will experience a higher quality of care, which will make them feel and express gratitude to the nurses that care for them; when nurses feel their work is recognized, they will feel more satisfied with their nursing work and their psychological health will be enhanced. ${ }^{[30]}$

\subsubsection{Implementation of specialized nurses in isolation wards}

In our study, many frontline nurses reported that the new intensive workload decreased their family time, which negatively affected their psychological health. China is currently training specialized nurses who can be added to nursing teams and decrease the high workload of current frontline nurses. ${ }^{[31]}$ For example, the ICU specialized nurses and respiratory specialized nurses can evaluate the pulmonary function of patients and provide appropriate respiratory support, allowing expedient ventilator removal. On the other hand, rehabilitation specialist nurses can teach patients breathing exercises, which will help improve their lung function. Psychological specialized nurses can help patients address psychological problems, so that patients afflicted with a negative mood can regain their spirit, cooperate with the treatment and overcome COVID-19. Specialized nurses can also improve access to treatment, which can ensure that a greater number of patients infected with COVID-19 are treated accordingly. ${ }^{[32]}$ The specialized nurses in isolation wards can perform their respective duties, improve the quality of nursing services, and provide patients with the most professional and refined nursing services. Furthermore, the implementation of specialized nurses can decrease the workload of current frontline nurses, which can prevent burnout and in- 
crease their psychological health by allowing them to spend more time with their families.

\subsubsection{Encouraging hospital managers to check-in with frontline nurses}

Nursing managers should pay attention to the work and life needs of frontline nurses to ensure they feel cared for, which can improve their psychological health. The isolation ward head nurse should regularly express gratitude and greet nurses in his division every week to learn about the current problems and solve them in a timely manner. Isolation ward nursing managers should work with nurses to schedule shifts that meet the scheduling requirements of nurses as much as possible. Nursing managers should improve communication with the logistics department, enhance the daily supply of food and isolation ward nurse necessities, and ensure that nurses have good physical quality to meet challenges. ${ }^{[33]}$ These changes should increase the physical and psychological health of frontline nurses.

\subsection{Reduce the negative factors affecting the psycholog- ical health of nurses}

\subsubsection{Address the family issues of nurses in isolation wards}

In our study, isolation ward nurses felt disconnected from their families because they worked long hours, which precluded them from spending quality time with loved ones. Decreased family time can increase the psychological burden experienced by isolation ward nurses, since perceived familial support is associated with positive affect and better mental health. ${ }^{[34]}$ Decreasing the worries of isolation ward nurses allows them to wholeheartedly devote themselves to their patients during the pandemic. ${ }^{[12,13]}$ In Wenzhou city, the hospital trade union outlined several difficulties faced by the families of isolation ward nurses and called on other hospital staff to help frontline healthcare workers solve the problems encountered in their family life. The municipal education bureau has also launched a voluntary service program called "School Companions During the Pandemic" to help children of medical personnel work on their schoolwork. The municipal government and local communities also arranged for staff to visit the families of frontline health care workers and provide one-on-one support. The implementation of such initiatives can help support the families of frontline workers, so nurses can rest assured that their loved ones are taken care of even in their absence, which can contribute to positive psychological health.

\subsubsection{Reduce the negative effects of protective equipment on nurses}

Due to the high infectivity of COVID-19, medical staff must wear protective equipment to enter the isolation ward. How- ever, interviewed nurses stated that daily use of this protective equipment causes them discomfort, and even physical pain. These findings align with those of previous studies highlighting the discomfort that protective equipment causes nurses working during the COVID-19 and SARS pandemics. ${ }^{[35]}$ For example, N95 masks cause pressure damage on the face, and goggles fog and occlude vision, which affect the efficiency of nurses and increases their perceived pressure. ${ }^{[36]}$ Some strategies can be used to reduce the side effects of protective equipment. For example, to reduce fogging of the goggles, antibacterial hand sanitizer and a layer of alcohol can be applied to the inside of the goggles with cotton swabs. After drying them, goggles could then be distributed to staff. Facial pressure injuries due to paste hydrocolloids from the facial masks and goggles can be reduced by moisturizing facial skin several times before pasting the hydrocolloids. Discomfort caused by hydrocolloids can also be avoided by using a viscose peel agent. ${ }^{[37,38]}$ Application of these strategies to the protective equipment of current COVID-19 frontline nurses can reduce their discomfort, thereby increasing their psychological health, and their abilities to tend to their patients.

\subsection{Limitations and future directions}

Our study used a qualitative phenomenological research framework to conduct interviews with frontline clinical nurses and analyze emerging themes. The study imposed strict inclusion criteria by only interviewing COVID-19 frontline clinical nurses, which made it representative of the population of interest. However, a limitation of this study is that only nurses were interviewed, not nursing managers or other hospital organizational personnel. Therefore, only the lived-experience perspective of clinical COVID-19 frontline nurses was obtained, not an institutional perspective. Future studies could evaluate the experiences of nursing managers and other hospital organizational personnel to make stronger recommendations on the institutional level.

\section{Conclusion}

In this study, in-depth interviews with 10 first-line clinical nurses in isolation wards during the COVID-19 pandemic were conducted. Three themes emerged: the evolution of psychological health; positive factors that affect psychological health; and negative factors that affect psychological health. Nurses experienced various psychological states during the pandemic. Appreciation from patients, support from team members, and feeling cared for by their organizations enhanced nurses' psychological health. Insufficient family support and discomfort from protective equipment negatively impacted nurses' psychological health. It is recommended that changes in the psychological health of isolation ward 
nurses should be constantly monitored. Proper strategies should be implemented to reduce the physical discomfort experienced by nurses due to protective equipment and increase perceptions of familial and workplace support. Nursing managers and nursing organizations should also explore means allowing nurses to feel appreciated by their patients.

\section{FUNDING}

Foundation of Zhejiang Education Committee (Y202044376)

\section{CONFlicts OF InTEREST Disclosure}

The authors declare that there is no conflict of interest.

\section{REFERENCES}

[1] World Health Organization. Coronavirus disease. 2020; Available from: www . who.int/emergencies/diseases/novel-coronav irus-2019

[2] Yuan C. A simple model to assess Wuhan lock-down effect and region efforts during COVID-19 epidemic in China Mainland. medRxiv, 2020.

[3] Chen S, et al. COVID-19 control in China during mass population movements at New Year. The Lancet. 2020; 395(10226): 764-766. https://doi.org/10.1016/S0140-6736(20)30421-9

[4] AlTakarli NS. China's Response to the COVID-19 Outbreak: A Model for Epidemic Preparedness and Management. Dubai Medical Journal. 2020: p. 1-6. https : //doi .org/10.1159/000508448

[5] Oh N, et al. Exploring Nursing Intention, Stress, and Professionalism in Response to Infectious Disease Emergencies: The Experience of Local Public Hospital Nurses During the 2015 MERS Outbreak in South Korea. Asian Nurs Res (Korean Soc Nurs Sci). 2017; 11(3): 230-236. PMid:28991605 https://doi.org/10.1 016/j.anr.2017.08.005

[6] Giarratano G, et al. Disaster research: a nursing opportunity. Nurs Inq. 2014; 21(3): 259-68. PMid:23899191 https://doi .org/10 $.1111 /$ nin. 12049

[7] Ruotsalainen JH, et al. Preventing occupational stress in healthcare workers. Cochrane Database of Systematic Reviews. 2014 https://doi.org/10.1002/14651858.CD002892.pub4

[8] Birhanu M, et al. Workload Determines Workplace Stress among Health Professionals Working in Felege-Hiwot Referral Hospital, Bahir Dar, Northwest Ethiopia. Journal of Environmental and Public Health. 2018. PMid:30598668 https://doi .org/10.1155/2018 16286010

[9] Chen CS, et al. Psychological distress of nurses in Taiwan who worked during the outbreak of SARS. Psychiatric Services. 2005; 56(1): 76-79. PMid:15637196 https ://doi .org/10.1176/appi .ps.56.1.76

[10] Wong TW, et al. The psychological impact of severe acute respiratory syndrome outbreak on healthcare workers in emergency departments and how they cope. European Journal of Emergency Medicine. 2005. 12(1): 13-18. PMid:15674079 https://doi .org/10.1097/0006 3110-200502000-00005

[11] Tam CW, et al. Severe acute respiratory syndrome (SARS) in Hong Kong in 2003: stress and psychological impact among frontline healthcare workers. Psychological Medicine. 2004; 34(7): 1197. PMid:15697046 https : //doi .org/10.1017/S0033291704002 247

[12] Koinis A, et al. The impact of healthcare workers job environment on their mental-emotional health. Coping strategies: the case of a local general hospital. Health Psychology Research. 2015; 3(1). PMid:26973958 https://doi.org/10.4081/hpr.2015.1984

[13] Hall LH, et al. Healthcare staff wellbeing, burnout, and patient safety: a systematic review. PloS one. 2016; 11(7): e0159015.
PMid:27391946 https://doi.org/10.1371/journal.pone.0 159015

[14] Niven K, Ciborowska N. The hidden dangers of attending work while unwell: A survey study of presenteeism among pharmacists. International Journal of Stress Management. 2015; 22(2): 207. https://doi.org/10.1037/a0039131

[15] Holden RJ, et al. Effects of mental demands during dispensing on perceived medication safety and employee well-being: A study of workload in pediatric hospital pharmacies. Research in social and administrative Pharmacy. 2010; 6(4): 293-306. PMid:21111387 https://doi.org/10.1016/j.sapharm.2009.10.001

[16] Shanafelt TD, et al. Burnout and medical errors among American surgeons. Annals of Surgery. 2010; 251(6): 995-1000. PMid:19934755 https://doi.org/10.1097/SLA.0b013e3181bfdab3

[17] Chen SHM, et al. Working in a danger zone: A qualitative study of Taiwanese nurses' work experiences in a negative pressure isolation ward. American Journal of Infection Control. 2016; 44(7): 809-14. PMid:26944003 https://doi.org/10.1016/j.ajic.2016.01 .023

[18] Happell B, et al. Nurses and stress: recognizing causes and seeking solutions. J Nurs Manag. 2013; 21(4): 638-47. PMid:23700980 https://doi.org/10.1111/jonm.12037

[19] Øye C, Sørensen NØ, Glasdam S. Qualitative research ethics on the spot: Not only on the desktop. Nursing Ethics. 2015; 23(4): 455-464. PMid:25665588 https://doi.org/10.1177/09697330145670 23

[20] Ghada Abu S. EMPLOYMENT OF COLAIZZI'S STRATEGY IN DESCRIPTIVE PHENOMENOLOGY: A REFLECTION OF A RESEARCHER. European Scientific Journal. 2012; 8(27).

[21] Su TP, et al. Prevalence of psychiatric morbidity and psychological adaptation of the nurses in a structured SARS caring unit during outbreak: a prospective and periodic assessment study in Taiwan. Journal of Psychiatric Research. 2007; 41(1-2): 119-130. PMid:16460760 https://doi.org/10.1016/j.jpsychires.2005.12.006

[22] Sun N, et al. A qualitative study on the psychological experience of caregivers of COVID-19 patients. American Journal of Infection Control. 2020.

[23] Khee KS, et al. The psychological impact of SARS on health care providers. Critical Care and Shock. 2004; 100-106.

[24] Klatt M, Steinberg B, Duchemin AM. Mindfulness in Motion (MIM): An Onsite Mindfulness Based Intervention (MBI) for Chronically High Stress Work Environments to Increase Resiliency and Work Engagement. J Vis Exp. 2015(101): e52359.

[25] Surbakti S, Novieastari E, Nuraini T. Caring efficacy to improve nurses' caring behavior. Enfermería Clínica. 2019; 29: 698702. PMid:31302023 https://doi.org/10.1016/j.enfcli.2 019.04 .107

[26] McCabe T, Sambrook S. The antecedents, attributes and consequences of trust among nurses and nurse managers: A concept analysis. International Journal of Nursing Studies. 2014; 51(5): 815-827. 
PMid:24182730 https://doi.org/10.1016/j.ijnurstu. 201 3.10 .003

[27] Morsiani G, Bagnasco A, Sasso L. How staff nurses perceive the impact of nurse managers' leadership style in terms of job satisfaction: a mixed method study. J Nurs Manag. 2017; 25(2): 119-128. PMid:27917561 https://doi.org/10.1111/jonm. 12448

[28] Kourkouta L, Papathanasiou IV. Communication in nursing practice. Materia Socio-Medica. 2014; 26(1): 65. PMid:24757408 https: //doi.org/10.5455/msm.2014.26.65-67

[29] Kelly LA, Lefton C. Effect of meaningful recognition on critical care nurses' compassion fatigue. American Journal of Critical Care. 2017; 26(6): 438-444. PMid:29092866 https://doi .org/10.4037/aj cc2017471

[30] McNicholas A, et al. Improving Patient Experience Through Nursing Satisfaction. J Trauma Nurs. 2017; 24(6): 371-375. PMid:29117055 https://doi.org/10.1097/JTN.0000000000000328

[31] Wong FKY. Development of advanced nursing practice in China: Act local and think global. International Journal of Nursing Sciences. 2018; 5(2): 101-104. PMid:31406809 https://doi.org/10.101 6/j.ijnss.2018.03.003

[32] Prevos-Morgant M, et al. Benefits of the epilepsy specialist nurses (ESN) role, standardized practices and education around the world.
Revue Neurologique. 2019; 175(3): 189-193. PMid:30683450 https://doi.org/10.1016/j.neurol.2018.10.003

[33] Maben J, Bridges J. Covid-19: Supporting nurses' psychological and mental health. J Clin Nurs. 2020.

[34] Thomas PA, Liu H, Umberson D. Family relationships and wellbeing. Innovation in Aging. 2017; 1(3): igx025. PMid:29795792 https://doi.org/10.1093/geroni/igx025

[35] Verbeek JH, et al. Personal protective equipment for preventing highly infectious diseases due to exposure to contaminated body fluids in healthcare staff. Cochrane Database of Systematic Reviews. 2020.

[36] Huang H, et al. COVID-19: A Call for Physical Scientists and Engineers. ACS NANO. 2020; 14(4): 3747-3754. PMid:32267678 https://doi.org/10.1021/acsnano.0c02618

[37] Douglas D, Douglas R. Addressing the corona virus pandemic: will a novel filtered eye mask help? International Journal of Infectious Diseases. 2020; 95: 340-344. PMid:32334119 https: //doi.org/10.1016/j.ijid.2020.04.040

[38] Li JO, et al. Novel Coronavirus disease 2019 (COVID-19): The importance of recognising possible early ocular manifestation and using protective eyewear. Br J Ophthalmol. 2020; 104(3): 297-298. PMid:32086236 https ://doi .org/10.1136/bjophthalmol-2 020-315994 